\title{
Retroperitoneal Laparoscopic Surgery in the Treatment of Complex Adrenal Tumors
}

This article was published in the following Dove Press journal:

Cancer Management and Research

\author{
Kai Huang (D) \\ Yehua Wang' \\ Xiao $\mathrm{Gu}^{\prime}$ \\ Qin Xiao ${ }^{2}$ \\ Xiangan $\mathrm{Tu}^{3}$ \\ 'Department of Urology, College of \\ Clinical Medicine, Yangzhou University, \\ Northern Jiangsu People's Hospital, \\ Yangzhou, Jiangsu Province, People's \\ Republic of China; ${ }^{2}$ Department of \\ Pathology, College of Clinical Medicine, \\ Yangzhou University, Northern Jiangsu \\ People's Hospital, Yangzhou, Jiangsu \\ Province, People's Republic of China; \\ ${ }^{3}$ Department of Urology and Andrology, \\ The First Affiliated Hospital of Sun Yat- \\ Sen University, Guangzhou, Guangdong \\ Province, People's Republic of China
}

Purpose: To explore the laparoscopic technique with the retroperitoneal approach for complex adrenal tumors.

Patients and Methods: The clinical data of 11 patients with complex adrenal tumors from July 2017 to July 2018 were analyzed retrospectively. Among them, there were 4 males and 7 females, 4 with adrenal myelolipomas, 3 with adrenal pheochromocytomas, 2 with adrenal cysts, 1 with adrenocortical adenoma and 1 with adrenal ganglioneuroma. The average tumor diameter was $6.5 \pm 1.2 \mathrm{~cm}$, and the average age of the patients was $48 \pm 13$ years.

Results: All the operations were successfully completed. The average operation time was 95 $\pm 15 \mathrm{~min}$, the average amount of blood loss was $50 \pm 15 \mathrm{~mL}$, and the average postoperative hospital stay was $2.6 \pm 1.3$ days. No tumor recurrence was found after 1 year of follow-up. Conclusion: Retroperitoneal laparoscopic surgery is effective for the treatment of complex adrenal tumors, but it requires good surgical skills. Surgeons skilled in laparoscopic technology can safely carry out retroperitoneal laparoscopic surgery for complex adrenal tumors.

Keywords: laparoscopy, retroperitoneal approach, complex adrenal tumor, surgery

\section{Introduction}

Surgery is the main method of treatment for adrenal tumors. In 1992, Gagner first reported transabdominal laparoscopic surgery for Cushing syndrome. ${ }^{1}$ With the progress of laparoscopic surgery technology and the improvement of equipment, laparoscopic surgery for adrenal diseases has been widely used by urologists. Because of the small space of the retroperitoneum, laparoscopic surgery for the treatment of complex adrenal tumors requires a highly skilled technique. There are few reports regarding retroperitoneal laparoscopic surgery for complex adrenal tumors. From July 2017 to July 2018, we treated 11 patients with this kind of disease, and we share our surgical experience.

\section{Patients and Methods}

From July 2017 to July 2018, 11 patients with complex adrenal tumors were treated via the retroperitoneal approach of laparoscopic surgery in our hospital. There were 7 female patients and 4 male patients with an average age of $(48 \pm 13)$ years. The tumor was located on the left adrenal gland in 7 patients and on the right in 4 patients. The diameter of the tumor was $3.5 \sim 13 \mathrm{~cm}$, with an average of $(6.5 \pm 1.2) \mathrm{cm}$. There were 4 patients with adrenal myelolipoma, 3 with adrenal pheochromocytoma, 2 with an adrenal cyst, 1 with adrenocortical adenoma and 1 with recurrent adrenal ganglioneuroma. The levels of serum cortisol (detected at 0:00 am, 8:00 am and 4:00 
pm, respectively), renin-angiotensin (in both the standing and supine positions), serum aldosterone and catecholamine were examined in all patients before the operation. Before the operation, plain and enhanced CT scans of the upper abdomen and chest were performed to evaluate whether the tumor was benign or malignant, where there were adhesions to the abdominal aorta, inferior vena cava or other large vessels, whether there were boundary outlines and whether there was surrounding tissue invasion. The surgical patients with pheochromocytoma were treated with $\alpha$-blocker drug that 10mgphenobenzamine was taken twice a day for 2 weeks before the operation, and blood pressure and heart rate were controlled to fully prepare for volume expansion.

General anesthesia was used during the operation. The patient was placed in the lateral position with 90 degrees on the raised lumbar bridge, and four trocars $(12,12,5$ and $5 \mathrm{~mm}$ ) were located at the waist. The first trocar was located at the junction of the posterior axillary line of the inferior edge of the twelfth rib, the skin was cut open, and the vascular clamp was used to bluntly penetrate into the retroperitoneal space through the lumbar dorsal fascia. After dilatation with a homemade air bag for 5 minutes, the trocar was inserted with the lens for monitoring. The other trocars were disposed $2 \mathrm{~cm}$ above the anterior superior iliac crest, at the junction of the anterior axillary line and the inferior costal arch, and at the horizontal umbilical cord of the anterior axillary line. After cleaning the extraperitoneal fat, the lateral cone fascia and renal fascia were opened to expose the adrenal tumor (Figure 1A), reducing intraoperative bleeding and retaining part of the fat around the adrenal tumor. During the operation, attention was paid to the exposure of the central adrenal vein. An ultrasonic scalpel combined with HEMO-LOCK and titanium clips was used to separate and expose the adrenal tumor to stop the bleeding completely, and attention was paid to avoid

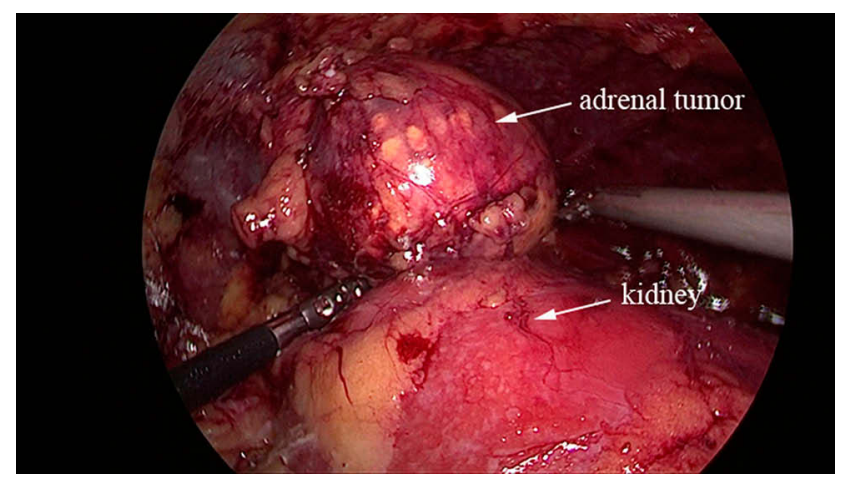

Figure I Exposure of adrenal myelolipoma. damaging the abdominal aorta, inferior vena cava and renal vein. The tumor was collected completely in the specimen bag and removed. The retroperitoneal silicone drainage tube was routinely placed, and the incision of the skin was sutured. The vital signs of the patients were monitored after the operation. Antibiotics were not used (only if the operation time was more than 2 hours and there was more blood loss during the operation were antibiotics temporarily used during the operation).

\section{Results}

In all 11 patients, the operations were completed successfully, and no patient required conversion to an open operation. Before the operation, the anesthesiologist performed radial artery and central vein catheterization to monitor the arterial and venous pressures. The individual operation time was $65 \sim 125$ minutes, and the amount of blood loss during the operation was $20 \sim 180 \mathrm{~mL}$. During the operation, there were 3 patients with peritoneal rupture and 1 patient with diaphragm injury, and none of the patients received blood transfusions. Adrenocorticoid supplementation was used appropriately to address the adrenal crisis caused by adrenocorticoid deficiency after the operation. Early activity was encouraged in all patients after surgery, and the drainage tube was removed as soon as possible. The postoperative hospital stay was 2 to 6 days, with an average of (2.6 \pm 1.3 ) days. During the operation, blood pressure fluctuations occurred in 3 patients, and the highest blood pressure reached 239/112 $\mathrm{mmHg}$ in 1 patient (Figure 2A). The operation was suspended temporarily. After anesthetic treatment and intervention, the operation was then carried out. The postoperative pathological results showed that there were 4 cases of adrenal myelolipoma, 3 cases of adrenal pheochromocytoma (Figure 2B and C), 2 cases of adrenal cyst, 1 case of adrenocortical adenoma and 1 case of recurrent adrenal ganglioneuroma (Figure 3). The patients were followed for 1 year, and no tumor recurrence was found by B-ultrasound, CT scan or other imaging examinations.

\section{Discussion}

Laparoscopic surgery has the characteristics of a clear field of vision, small incision and limited bleeding. Operative complications can be effectively reduced. Postoperative pain is mild, and patients can return to activity early as soon as possible. The operative approach for laparoscopic adrenal tumors can be completed by transabdominal and retroperitoneal approaches. Surgery via the transabdominal 


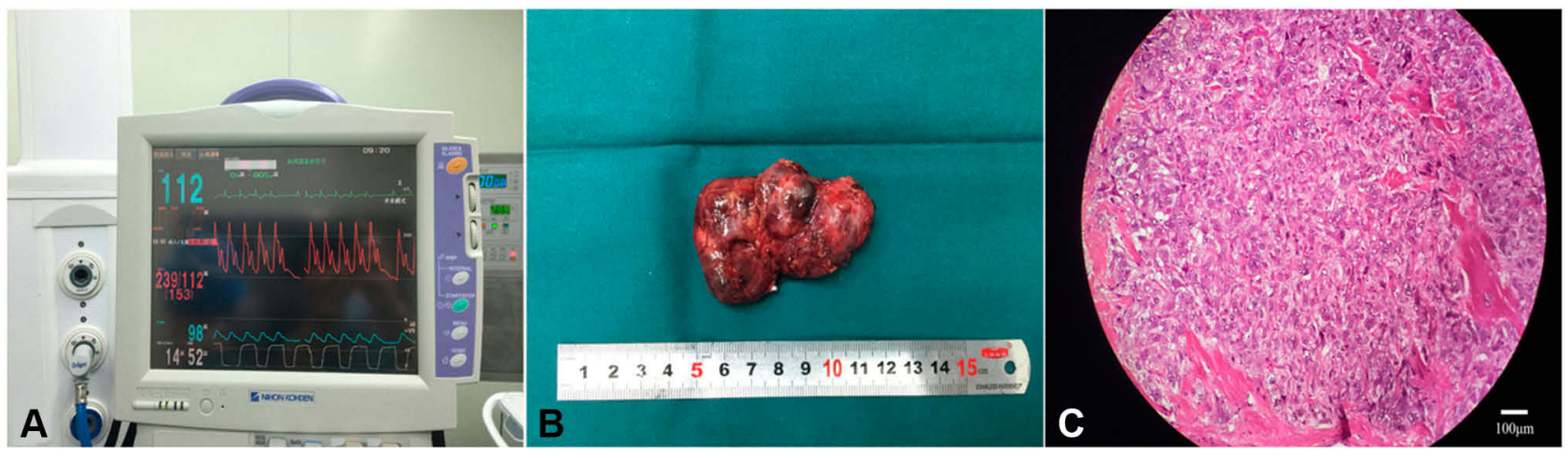

Figure 2 (A). During the operation for adrenal pheochromocytoma, the blood pressure reached as high as $239 / 112 \mathrm{mmHg}$; (B). Postoperative mass specimen of adrenal pheochromocytoma; (C). Pathological image of adrenal pheochromocytoma with hematoxylin and eosin staining, the bar is $100 \mu \mathrm{m}$.

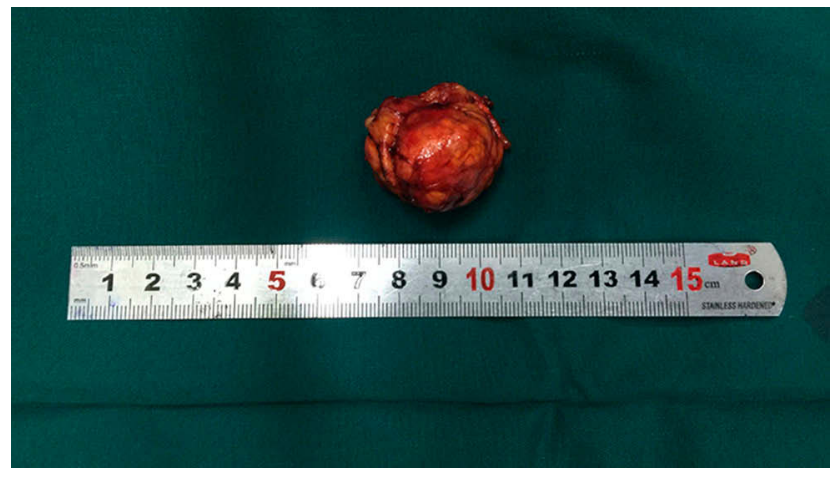

Figure 3 Postoperative mass specimen of recurrent adrenal ganglioneuroma.

approach can obtain a large operating space, and the anatomical marks can be clearly identified. ${ }^{2}$ Although the operation space of retroperitoneal approach is small, it can still identify the anatomical signs such as psoas major muscle, perirenal fascia, peritoneum and kidney and so on through the accumulation of surgical experience, to fully expose the adrenal area for adrenalectomy. Some researchers ${ }^{3}$ believe that the retroperitoneal approach can avoid abdominal stimulation and reduce the probability of intestinal injury. For small adrenal tumors $(\square 2 \mathrm{~cm}$ ), the retroperitoneal approach is generally chosen for laparoscopic adrenal surgery. However, for complex adrenal tumors, there are few reports on retroperitoneal laparoscopic surgery. Referring to relevant research reports, ${ }^{4-6}$ tumors with the following characteristics are defined as complex adrenal tumors: (1) large adrenal tumors with a general diameter over $6 \mathrm{~cm}$; (2) adrenal pheochromocytoma; (3) adrenal malignant tumors; (4) bilateral adrenal tumors; (5) adrenal tumors with a previous history of surgery on peri-adrenal organs; and (6) obese patients (body mass index $>25$ ). For adrenal tumors whose diameter is larger than $6 \mathrm{~cm}$, some studies ${ }^{7}$ believe that laparoscopic resection of complex adrenal tumors is associated with an insufficient operating space. Intraoperative bleeding is not easy to control, and it can easily cause damage to adjacent organs of the adrenal gland. In our study, 4 cases of adrenal myelolipoma and 2 cases of adrenal cyst were larger than $6 \mathrm{~cm}$ in diameter. In some cases, the diameter of the adrenal mass reached $13 \mathrm{~cm}$, and the space between the adrenal mass and surrounding organs was small. Thus, added caution and high surgical skills are required during the operation. Because the operation has no significant effect on blood pressure or heart rate, attention had been paid to the treatment of adrenal vasculature. Laparoscopic surgery can still be performed through retroperitoneal surgery for complex adrenal tumors with diameters larger than $6 \mathrm{~cm}$. We believe that during the operation, the retroperitoneal space should be expanded as much as possible, and unnecessary perirenal fat should be removed. The range of free exposure should be from the diaphragm to the inferior pole of the kidney and from the upper pole to the extraperitoneal part of the liver or splenic area. It is important to maintain the integrity of the peritoneum in the operative space. If necessary, excess adrenal fat can be removed to expose the surgical field of vision.

CT enhanced scans and MRI examinations should be performed before surgery, and CTA angiography should be performed if possible. ${ }^{8}$ Radiologists should be consulted to determine the location of the tumor and its adjacent relationship to surrounding organs and blood vessels. Most complex adrenal tumors are more than $6 \mathrm{~cm}$ in diameter and are often accompanied by enlarged central adrenal veins. Therefore, the blood supply of complex adrenal tumors is relatively abundant, and the arterial branches of 
these tumors can be supplied by branches of the abdominal aorta and renal artery. ${ }^{9}$ Therefore, it is of great significance to clarify the blood supply of adrenal tumors for retroperitoneal laparoscopic surgery.

Vascular injury is the most common complication of laparoscopic adrenal surgery, ${ }^{10}$ and it is more likely to occur in complex adrenal tumor surgery. In most cases, vascular injury is due to unclear exposure to the vascular structure, resulting in acute injury or burns. Generally, there is a greater chance of inferior vena cava injury. When dissociating the tumor, it cannot be lifted and dissociated directly due to the large size of the tumor. Some studies ${ }^{11}$ suggest that the tissue around the tumor can be clamped, and the tumor can be flipped and dissociated from different sides. When we perform the operation, we first dissociate the dorsal, ventral, superior and inferior poles of the adrenal tumor and finally dissociate the medial part of the adrenal tumor to deal with the central vein of the adrenal gland. In one patient in this group, the diameter of the adrenal pheochromocytoma was $4.8 \mathrm{~cm}$, and the tumor had a certain adhesion to the psoas major muscle and base of the diaphragm. During the operation, we tried our best to expose the local operation field, and the renal vein and inferior vena cava were exposed in the operation field. The operation can be carried out successfully by controlling the blood supply of the tumor as soon as possible. We believe that anatomic structures should be carefully identified during the operation, especially in the case of amputation. The tissue around the tumor should not be damaged, and the tumor vessels should be ligated in time to avoid the unclear surgical field affecting the operation.

Preoperative preparation for patients with complex adrenal tumors is very important. A-receptor blocking drugs are used to control hypertension before surgery in patients with pheochromocytoma. If the control of blood pressure is not satisfactory, calcium channel blocking drugs can be added to stabilize the blood pressure at approximately $120 / 80 \mathrm{mmHg} .{ }^{12}$ For patients with arrhythmia, $\beta$-blocker drugs can be added to keep the heart rate as low as 90 beats/min after the use of $\alpha$-blocker drugs. During the operation, the key point is to dissociate the surrounding tissue of the pheochromocytoma gently to avoid excessive violence to squeeze the tumor. Especially when clamping the adrenal central vein, we should communicate with anesthesiologists to facilitate the timely use of drugs to ensure the stability of hemodynamics. This is helpful for the safe completion of the operation.

\section{Conclusion}

Laparoscopic surgery for complex adrenal tumors, especially for large adrenal tumors, is difficult because of insufficient space and exposure. The results of our study show that laparoscopic resection of complex adrenal tumors via a retroperitoneal approach is feasible and that the effect of the operation is good. We believe this technique can be accomplished with the accumulation of experience in laparoscopic surgery, with full evaluation of the condition before the operation, and with preparation for the risks that may occur during the operation. Of course, the accumulation of more cases of retroperitoneal laparoscopic surgery for complex adrenal tumors will help to explore the standard process of retroperitoneal laparoscopic surgery for complex adrenal tumors. However, it is also necessary to formulate a definite operative plan according to the actual situation of the patients and the experience of the operators. For some cases of adrenal tumors with local infiltration or invasion of large vessels, other treatment methods should be considered.

\section{Acknowledgments}

This study wasn't based on a previous communication to a society or meeting. This study was approved by the ethics committee of college of clinical medicine of Yangzhou University, Northern Jiangsu People's Hospital. Written informed consent was obtained from patients.

\section{Disclosure}

The authors report no funding and no conflicts of interest for this work.

\section{References}

1. Gagner M, Lacroix A, Bolté E. Laparoscopic adrenalectomy in cushing's syndrome and pheochromocytoma. $N$ Engl J Med. 1992;327 (14):1033. doi:10.1056/NEJM199210013271417

2. Fiori C, Checcucci E, Amparore D, Cattaneo G, Manfredi M, Porpiglia F. Adrenal tumours: open surgery versus minimally invasive surgery. Curr Opin Oncol. 2020;32(1):27-34. doi:10.1097/CCO.00000000 00000594

3. Zhu W, Wang S, Du G, Liu H, Lu J, Yang W. Comparison of retroperitoneal laparoscopic versus open adrenalectomy for large pheochromocytoma: A single-center retrospective study. World J Surg Oncol. 2019;17(1):111. doi:10.1186/s12957-019-1649-x

4. Wang Y, He Y, Li BS, et al. Laparoendoscopic single-site retroperitoneoscopic adrenalectomy versus conventional retroperitoneoscopic adrenalectomy in obese patients. $J$ Endourol. 2016;30(3):306-311. doi:10.1089/end.2015.0526

5. Agha A, Iesalnieks I, Hornung M, et al. Laparoscopic trans- and retroperitoneal adrenal surgery for large tumors. J Minim Access Surg. 2014;10(2):57-61. doi:10.4103/0972-9941.129943 
6. Maestroni U, Vicente D, Del Rio P, et al. Laparoscopic adrenalectomy for large adrenal masses: a challenge or a routine? Minerva Chir. 2014;69(2):59-64.

7. Liu H, Li B, Yu X, Huang Y. Perioperative management during laparoscopic resection of large pheochromocytomas: A single-institution retrospective study. J Surg Oncol. 2018;118(4):709-715. doi:10.1002/jso.25205

8. Farrugia FA, Martikos G, Tzanetis P, et al. Pheochromocytoma, diagnosis and treatment: review of the literature. EndocrRegul. 2017;51(3):168-181. doi:10.1515/enr-2017-0018

9. Giurazza F, Corvino F, Silvestre M, et al. Adrenal glands hemorrhages: embolization in acute setting. Gland Surg. 2019;8(2):115122. doi: $10.21037 /$ gs.2018.10.06
10. Christakis I, Ng CS, Chen C, et al. Operation duration and adrenal gland size, but not BMI, are correlated with complication rate for posterior retroperitoneoscopic adrenalectomy for benign diseases. Surgery. 2019;165(3):637-643. doi:10.1016/j.surg.2018.09.004

11. Horesh N, Jacoby H, Dreznik Y, et al. Teaching laparoscopic adrenalectomy to surgical residents. $J$ Laparoendosc Adv Surg Tech A. 2016;26(6):453-456. doi:10.1089/lap.2015.0625

12. Naranjo J, Dodd S, Martin YN. Perioperative Management of Pheochromocytoma. J Cardiothorac VascAnesth. 2017;31(4):14271439. doi:10.1053/j.jvca.2017.02.023

\section{Publish your work in this journal}

Cancer Management and Research is an international, peer-reviewed open access journal focusing on cancer research and the optimal use of preventative and integrated treatment interventions to achieve improved outcomes, enhanced survival and quality of life for the cancer patient.
The manuscript management system is completely online and includes a very quick and fair peer-review system, which is all easy to use. Visit http://www.dovepress.com/testimonials.php to read real quotes from published authors. 